\title{
Auditory concept identification as a function of subject sex and stimulus dimensions
}

\author{
Vladimir Pishkin and Robert J. Blanehard \\ VA HOSPITAL AND UNIVERSITY OF OKLAHOMA SCHOOL OF MEDICINE
}

\begin{abstract}
Binary stimulus dimensions of duration, laterality, frequency, amplitude, and number were compared for their respective information values in auditory concept identification of males and females. Amplitude, without irrelevant information, was shown to provide significantly less information than the other dimensions with the exception of number. Males performed significantly poorer than females with laterality relevant under conditions of 1,2 , and 3 irrelevant dimensions. Sex differences in laterality were replicated with additional Ss.
\end{abstract}

\section{Problem}

In concept identification (CI) with visual stimuli, learning has been found to be most rapid when color is the relevant dimension, and proceeds at essentially the same rate when number, position, size, or shape are relevant (Bourne \& Restle, 1959; Pishkin, 1960). While these various dimensions have been studied in visual $\mathrm{CI}$, no attempt has been made to compare the rate of information processing under various dimensions of the auditory CI as a function of sex. Bulgarella \& Archer (1962), in the single preceding study of auditory CI, have employed several stimulus dimensions with females only. They found a systematic increase in S's errors as the number of irrelevant dimensions increased. This finding was in general agreement with studies of Bourne (1957), Pishkin (1960), and Pishkin \& Wolfgang (1964) of visual CI.

The present investigation consists of a series of 3 experiments attempting to determine the saliency of 5 auditory dimensions and the function of irrelevant information and sex. In the first experiment the S was presented with an auditory signal varying in a binary fashion and required to categorize the stimulus. This was the simplest CI task, in which only one dimension is employed with no irrelevant information. In the second experiment irrelevant dimensions were introduced and CI was studied as a function of both the number of irrelevant cues and also as a function of the type of stimulus employed. The final experiment replicated a portion of the second, where difference between male and female performance was validated.

\section{Subjects}

Experiments 1, 2, and 3 employed 50, 54, and $30 \mathrm{Ss}$, respectively. The Ss were hospital employees at the University of Oklahoma Medical Center. They ranged in age from 25 to $40 \mathrm{yr}$. One half of the Ss in each group were male, the remainder female.

\section{Procedure}

The Ss were run in a soundproofed room and wore headphones throughout the experiment. In Experiment 1, the auditory dimensions employed were amplitude (30 or $60 \mathrm{db}$ ); duration (1 sec. or $3 \mathrm{sec}$.); number (1 or 2 repetitions of the stimulus complex); frequency $(1,000$ or $3,000 \mathrm{cps}$ ); and signal laterality (presentation of the stimulus complex on the right or the left headphone). The Ss were presented with a signal which varied in only one of these stimulus dimensions. Their task was to categorize one stimulus as an X stimulus, the other stimulus as an 0 stimulus. They were instructed to respond verbally by describing each signal as either an $\mathrm{X}$ or 0 signal. For example, $30 \mathrm{db}$ tones were $\mathrm{X}$ tones, while $60 \mathrm{db}$ tones were 0 tones, with amplitude relevant. Instructions to the S included a description of the stimulus dimensions employed and their levels; an explanation of the significance of the feedback lights and a request for the $\mathrm{S}$ to respond as rapidly as possible. After S's response the correct category was given by feedback lamps mounted $5 \mathrm{ft}$ from the S's chair. The post-feedback interval was $3 \mathrm{sec}$. in all conditions.

Experiment 2 employed 3 relevant stimulus dimensions, duration, laterality, and frequency. Laterality, duration, number and frequency were the irrelevant cues. The design of this experiment may be viewed as a $3 \times 3 \times 2$ factorial with 3 types of relevant dimensions employed, 3 degrees of irrelevant information (1,2 or 3 irrelevant dimensions), and S's sex as the 2 group variables. There were $9 \mathrm{Ss}$ in each of the 6 treatment groups, with collapsed conditions of 3 relevant dimensions which were found to be equivalent in Experiment 1.

In Experiment 3, laterality alone was the relevant dimension, coupled with either 1,2 , or 3 irrelevant dimensions. This experiment was a replication of the laterality portion of Experiment 2 due to the function of sex variable in respect to laterality.

\section{Results and Discussion}

Presented in Fig. 1 are the mean number of errors made by Ss in Experiment 1 for each of the 5 stimulus dimensions. The Ss identifying stimuli varying in frequency made fewer errors than with any other stimulus dimension. Identifying the two levels of stimulus amplitude resulted in more errors than any other stimulus dimension. Mann Whitney $U$ tests were used to compare the mean errors made by Ss performing under the various dimensions. Ss identifying stimuli varying in frequency made significantly fewer errors than those required to make such identifications on the basis of amplitude or laterality $(\mathrm{U}=12,14.5 ; \mathrm{P}<.05)$. Stimulus amplitude identification resulted in significantly more errors than identification of variations in duration, fre- 


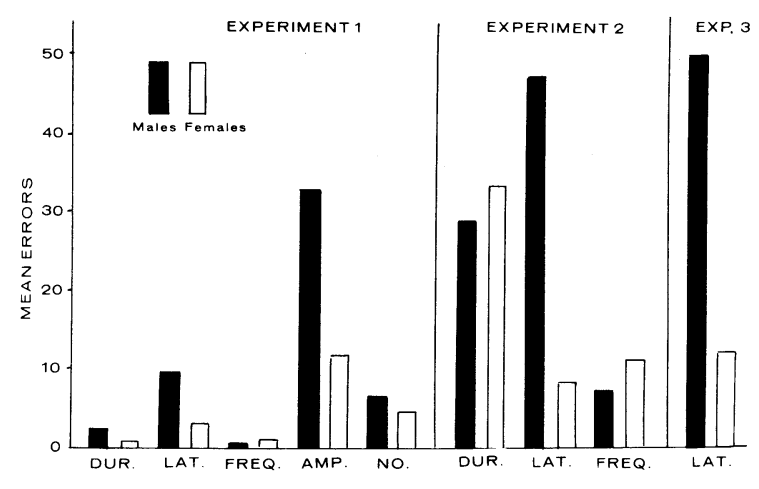

Fig. 1. Mean errors for males and females in 3 experiments. Note consistency of results for laterality between Experiments 2 and 3.

quency, or laterality $(\mathrm{U} \doteq 18.5,17.5,14.5 ; \mathrm{P}<.05)$. Figure 1 shows that for all conditions except frequency females tended to identify stimuli more rapidly. Statistical analysis, however, revealed no significant differences between sexes for each dimension.

Experiment 2 introduces irrelevant information by employing either 1, 2, or 3 irrelevant dimensions. Figure 1 presents the mean number or errors for Ss having 1, 2 , or 3 irrelevant dimensions present on each trial. As in Experiment 1, frequency as a relevant dimension tended to produce fewer errors than either laterality or duration. Males made significantly more errors than females in CI when laterality was the relevant stimulus dimension. Table 1 presents the data broken down by levels of complexity, sex, and type of dimension. Increasing numbers of irrelevant dimensions tended to produce more errors. At low levels of complexity males tended to make more errors when laterality was the relevant dimension than females. Due to the marked heterogeneity of variance, Mann Whitney Us were employed to compare dimensional irrelevant stimulus and sex differences. Two Us reached significance at the .05 level. First, Ss who were presented a stimulus consisting of 1 relevant dimension and 3 irrelevant dimensions made significantly more errors than those who had 1 relevant stimulus dimension and only 1 irrelevant stimulus dimension $(U=100 ; P<.05)$. Secondly, males again made significantly more errors than females when laterality was the relevant dimension $(\mathrm{U}=16 ; \mathrm{P}<.05)$.

Table 1. Mean Errors Produced by Males and Females under Conditions of Relevant and Irrelevant Dimensions

Relevant Dimensions

\begin{tabular}{crrrrrr}
\multicolumn{2}{l}{$\begin{array}{c}\text { No. Irrel. } \\
\text { Dimensions }\end{array}$} & \multicolumn{2}{c}{ Duration } & \multicolumn{2}{c}{ Laterality } & \multicolumn{2}{c}{ Frequency } \\
& & Females & Males & Females & Males & Females \\
& & & & & & \\
1 & 4.33 & 10.00 & 49.67 & 2.67 & 4.67 & 10.67 \\
2 & 10.33 & 10.33 & 51.67 & 8.00 & 13.33 & 13.33 \\
3 & 72.33 & 81.00 & 41.50 & 14.67 & 4.00 & 12.00
\end{tabular}

Figure 1 also shows the number of errors made by Ss in Experiment 3. Experiment 3 is identical to the laterality portion of Experiment 2. It may be seen that again males produced more errors than females when laterality was relevant. A test comparing male and female performance when laterality was the relevant dimension again was highly significant $(\mathrm{U}=38.5 ; \mathrm{P}<.001)$.

The relationship between rate of CI with no irrelevant information presented to the $\mathrm{S}$ and the stimulus amplitude is puzzling. Ss took significantly longer to categorize a signal on the basis of its amplitude than on the basis of any other variable except number. The major finding of Experiment 2 and Experiment 3 is that stimulus laterality as a relevant dimension in CI produces significantly faster learning for females as compared to males.This finding was replicated. While various studies have reported sex differences in frequency thresholds in audition (occupational factors), no previous study compared the propensity of males and females in the auditory localization (Beasley, 1938). This result tends to indicate that females localize sounds significantly easier than males.

The present investigation strongly indicates that auditory CI is influenced by the type of dimensions employed. While in visual CI on all stimulus cues except color are learned at essentially the same rate, in auditory CI the dimensions of amplitude and laterality tend to interact with either the sex of the $\mathrm{S}$ or provide less relevantutilizable information than number, frequency and duration. Since there is no known psychophysiological or neurological explanation for the present sex difference, it is plausible that the underlying factors may be of cultural or developmental nature. Possible changes in auditory sensitivity to laterality as a function of sex and chronological age should be explored.

\section{Referenees}

BEASLEY, W. C. National Health Survey, Hearing Study Series, Bulletin 5. The United States Public Health Service, 1938.

BOURNE, L. E., JR. Effects of delay of information feedback and task complexity on the identification of concepts. J. exp. Psychol., 1957, 54, 201-207.

BOURNE, L. E., JR., \& RESTLE, F. Mathematical theory of concept identification. Psychol. Rev., 1959, $66,278-296$.

BULGARELLA, R. G. , \& ARCHER, E. J. Concept identification of auditory stimuli as a function of amount of relevant and irrelevant information. J. exp. Psychol., 1962, 63, 254-257.

PISHKIN, V. Effects of probability of misinformation and number of irrelevant dimensions upon concept identification. J. exp. Psychol., 1960, 59, 371-378.

PISHKIN, V., \& WOLFGANG, A. Electromyographic gradients in concept identification with numbers of irrelevant dimensions. J. clin. Psychol., 1964, 20, 6167. 